\title{
THE LOCALIZATION OF STRAIN IN LOW SOLVUS HIGH REFRACTORY (LSHR) NICKEL SUPERALLOY
}

\author{
S.J. Kuhr ${ }^{1}$, G.B. Viswanathan², J. Tiley², H.L. Fraser ${ }^{1}$ \\ ${ }^{1}$ Materials Science Department, The Ohio State University, Columbus, OH \\ ${ }^{2}$ Air Force Research Laboratory, Wright Patterson AFB, OH
}

Keywords: Nickel base superalloy, EBSD, digital image correlation

\begin{abstract}
The low solvus high refractory (LSHR) nickel base superalloy was chosen as a candidate for a dual microstructure heat treatment (DMHT) turbine disk, with fine grains in the bore and coarser grains in the rim. In between those two regions, there is a transition in grain size and $\gamma^{\prime}$ precipitation. In this study, the mechanical performance of the coarse grain region was evaluated by room temperature tensile testing in the un-aged and dual aged conditions. Optical digital image correlation (DIC) was used to detect the localized strains evolving from the tensile testing. Strain maps from the DIC successfully detected localized strains in both of the microstructural conditions of interest. Transmission and scanning electron microscopy was use to analyze the deformed regions to assess grain misorientation and dislocation activity within each microstructure. While dislocations appeared to accumulate in the gamma channels in both microstructures, there appeared to more grain misorientation in the unaged condition of LSHR.
\end{abstract}

\section{Introduction}

The LSHR nickel base superalloy was developed to combine the low solvus of René 104 and the higher refractory content of Alloy 10 [1]. It was believed that with this combination of composition, that turbine disks would no longer suffer from quench cracking during fabrication [2]. The enhanced processing capability of this alloy made it a prime candidate for the dual microstructure heat treatment (DMHT) developed by NASA. The details of the DMHT can be found elsewhere $[1,3]$. The DMHT allows the turbine disk to optimize the strength dependent properties in the bore with fine grains as well as the time-dependent properties in the rim with coarser grains [1].

However, grain size is not the only concern with respect to the optimization of turbine disk mechanical properties. LSHR is a precipitate strengthened nickel base superalloy. Previous researchers have demonstrated the complex strengthening mechanisms that exist in such alloy systems [4-7]. They showed that the size, distribution and volume fraction of $\gamma^{\prime}\left(\mathrm{Ni}_{3} \mathrm{Al}\right)$ precipitates influence the behavior of linear and planar defects in the material and thus, greatly influence the mechanical performance of the turbine disk. Following the DMHT, the microstructure exhibited several modes of precipitation, including primary, secondary and tertiary distributions. Since the $\gamma^{\prime}$ phase has proven to be such a critical part of the strengthening, turbine disks are aged to stabilize the phase prior to use [8]. Aging studies performed by NASA [9] determined that a dual aging sequence provided the best balance in mechanical properties for the LSHR alloy. At the conclusion of that study, the process by which the secondary and tertiary $\gamma^{\prime}$ precipitates exchanged volume fractions remained unclear.
The objective of the current investigation was to determine the effect of the dual aging process on the mechanical response of the LSHR alloy. It is likely that various strengthening mechanisms are activated simultaneously to account for the macroscopic performance of the alloy [4]. For this reason, digital image correlation (DIC) techniques were used in an attempt to determine which microstructural features influence the localization of strain in the material. Previous studies have shown that when DIC techniques are combined with orientation information from electron backscatter diffraction (EBSD), valuable information about strain localization can be obtained [10].

In an attempt to simplify the microstructural features contributing to experimental observations, the coarse grains in the rim section of the disk were initially chosen for this investigation. This section of the disk has a more uniform microstructure compared with that of a gradient containing coarse and fine grains and lacks the micron sized primary $\gamma^{\prime}$ found in the bore section of the disk. The mechanical performance of the coarse grain microstructure was evaluated in the un-aged and dual aged condition with room temperature tensile testing. Strain maps were constructed of the sample surface using optical DIC techniques to detect the localization of strain. Strain localizations were further analyzed using scanning electron microscopy (SEM) and transmission electron microscopy (TEM) based techniques.

\section{Materials and Procedure}

The composition of the LSHR alloy is displayed in Table 1.

Table 1. Composition of LSHR (wt \%)

$\begin{array}{llllllllllll}\text { Co } & \mathrm{Cr} & \mathrm{Al} & \mathrm{Ti} & \mathrm{W} & \mathrm{Mo} & \mathrm{Ta} & \mathrm{Nb} & \mathrm{C} & \mathrm{B} & \mathrm{Zr} & \mathrm{Ni}\end{array}$ $\begin{array}{llllllllllll}20.4 & 12.3 & 3.5 & 3.5 & 4.2 & 2.7 & 1.6 & 1.5 & .05 & .03 & .05 & \text { Bal }\end{array}$

The LSHR superalloy was received after the DMHT was completed (without any additional aging). Subscale tensile specimens were extracted from the rim of the disk forging. Figure 1 displays the size and geometry of the specimens as well as and the location and orientation for extraction (relative to the turbine disk forging).

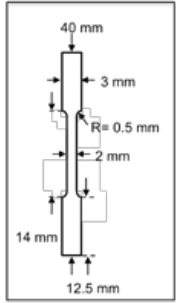

(a)

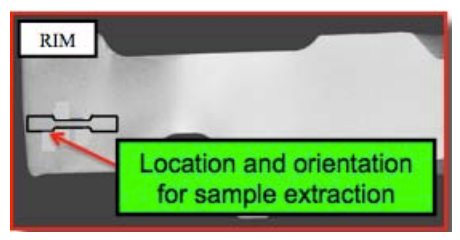

(b)
Figure 1. (a) Tensile specimen geometry used for mechanical testing (b) Location and orientation of sample extraction 
This sample geometry was specifically used for testing on an Electrothermal Mechanical Tester (ETMT). The ETMT, shown in Figure 2, is a modified load frame capable of simultaneously controlling load, displacement and temperature (via direct resistive heating) [11]. The ETMT used was developed by the UK National Physical Laboratory and Instron and is located at the Center for Accelerated Maturation of Materials (CAMM) at the Ohio State University.

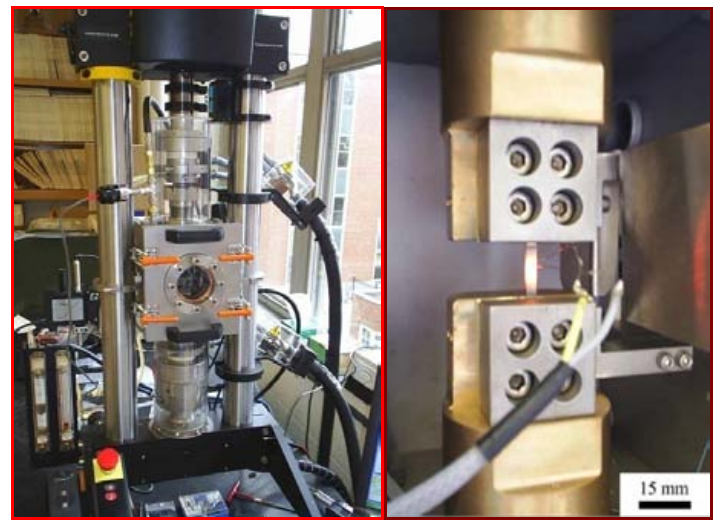

Figure 2. (a) Electrothermal Mechanical Tester (ETMT) at CAMM (b) A sample in the ETMT chamber being held at held at temperature for a mechanical test [11]

After extraction, one sample was left in the un-aged condition, while another was subjected to NASA's dual aging sequence. Before commencing with the heat treatment, the sample was glass encapsulated and backfilled with argon to prevent unwanted oxidation. The two-step aging sequence consisted of an initial exposure of $860^{\circ} \mathrm{C}$ for 4 hours with a furnace cool, followed by $780^{\circ} \mathrm{C}$ for 8 hours with an air cool. Room temperature tensile tests were performed using the ETMT under a constant strain rate of $8.3 \times 10^{-5} \mathrm{~s}^{-1}$

Digital image correlation software (VIC-2D) was used to determine the total amount of strain in the sample and to generate strain maps of localized strain intensity. Using this technique, two general assumptions are made: (1) there is a direct correspondence between the motion of points in the collected images and motion of points on the object and (2) that there is adequate spatial contrast to define and track local motions [12]. A random paint speckle pattern was applied to the sample surface to optimize the local spatial contrast required for the correlation functions in the software [12]. An example of an experimental speckle pattern can be seen in Figure 3. The vertical line through the center of the gage section in the image displays the digital extensometer used in the software to calculate the total strain in the sample. Further details of the mathematics and experimental procedures for using DIC can be found in the reference by Sutton [13].

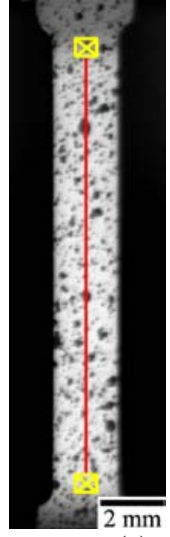

(a)

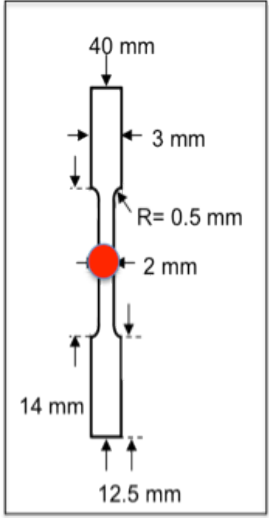

(b)
Figure 3. (a) Optical image of speckle pattern required for image correlation. The vertical line on the image displays the digital extensometer (b) Location microstructural examination on the tensile specimen, illustrated with the circle in the center of the gage section

Field emission scanning electron microscopy (SEM) [14] was used to observe the $\gamma^{\prime}$ volume fractions in each of the microstructures of interest. Before examining samples in the SEM, they were initially prepared with standard metallographic techniques and finished with $0.05 \mu \mathrm{m}$ colloidal silica. Since the atomic number contrast is typically poor between the' jand $\gamma$ phases [14], the samples were etched with a solution that contained $50 \mathrm{ml}$ of lactic acid, $30 \mathrm{ml}$ of nitric acid, and $2 \mathrm{ml}$ of hydrofluoric acid. The etchant is designed to remove the $\gamma$ phase, thus enhance the contrast during imaging.

A MATLAB based image processing software program developed at CAMM was used to determine the volume fraction and equivalent diameters of the $\gamma^{\prime}$ precipitates in the high-resolution SEM images. As a point of reference, $\gamma^{\prime}$ greater than $100 \mathrm{~nm}$ will be referred to as "secondary" and less than $100 \mathrm{~nm}$ as "tertiary" [9]. Electron backscatter diffraction (EBSD) measurements were made to determine the grain misorientations due to deformation. The misorientations were determined using the EDAX TSL Orientation Image Microscopy (OIM) software.

Focused Ion Beam (FIB) foils were extracted from each of the microstructures. This method of TEM sample preparation is particularly useful for site-specific sample extraction as required in this investigation. FIB foils were extracted from areas of highly localized strain, which were detected using the VIC-2D software. Bright field (BF) scanning transmission electron microscopy (STEM) was performed to observe dislocation activity within the deformed regions. According to Phillips [15], BF STEM zone axis imaging simultaneous uses multiple diffraction vectors such that any arbitrary lattice defect should exhibit contrast. Thus, zone axis imaging allows for a broad view of the existing defects in the microstructure and was pursued in this investigation for the $<110>$ zone axis. 


\section{Results and Discussion}

Heat Treatment

As a point of reference, the microstructures in the center of each tensile specimen (shown in Figure 3b) were initially compared for differences in grain and precipitate size and distribution. Figure 4 displays the inverse pole figure (IPF) maps generated from EBSD measurements of the central regions of the un-aged and dual aged tensile specimens. The maps show the grain size and orientation in which neither sample appeared to be highly textured.

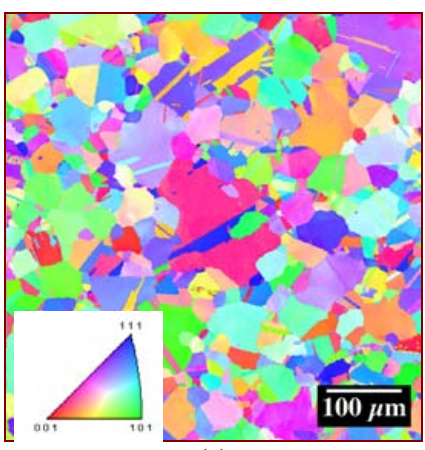

(a)

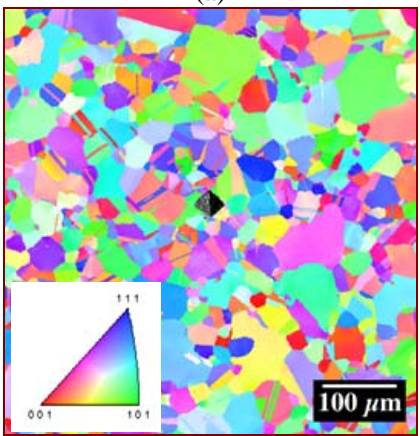

(b)

Figure 4. IPF maps of (a) un-aged (b) dual aged LSHR (in this case a hardness indent was used to mark the location of interest)

Figure 5 displays high-resolution SEM images of the un-aged and dual aged LSHR microstructures treated with the gamma prime etchant. Measurements of the volume fractions of the $\gamma^{\prime}$ particles as a function of etching time were performed. The results prove to be reliable and repeatable for etching times between 3 to 5 seconds [16]. Both microstructures appear to have a bimodal distribution of $\gamma^{\prime}$ and display secondary $\gamma^{\prime}$ as rounded cuboids. One difference that immediately appears is the dispersion of tertiary precipitates. Higher magnifications (displayed in Figure 5(c) and 5(d)) of the microstructure show that the there is no longer a fine dispersion of tertiary gamma prime in the material after the dual aging sequence.

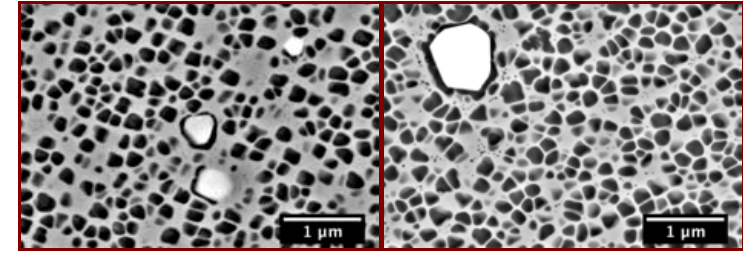

(a)

(b)

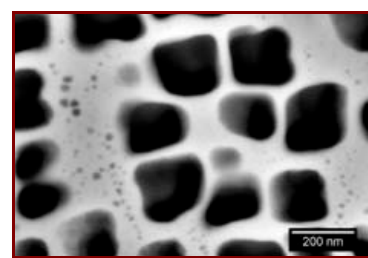

(c)

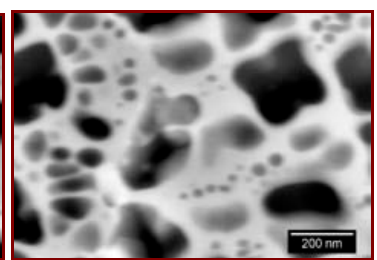

(d)
Figure 5. High resolution SEM images of (a) unaged microstructure (b) dual aged microstructure (c) higher magnification of unaged microstructure (d) higher magnification of dual aged microstructure

Stereological measurements of the microstructure were pursued to further quantify the differences between the microstructures. Segmentation routines were performed using the MATLAB based software developed at CAMM. Figure 6 displays the thresholded binary images of the $\gamma^{\prime}$ precipitates.

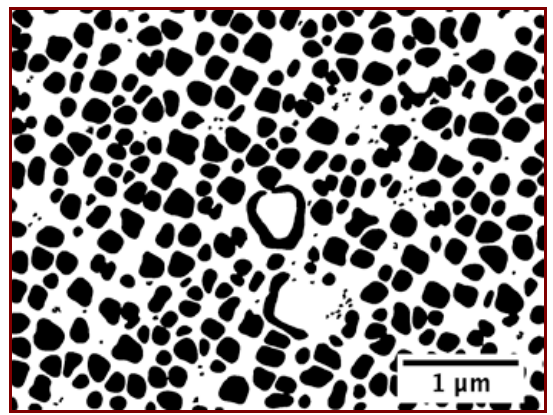

(a)

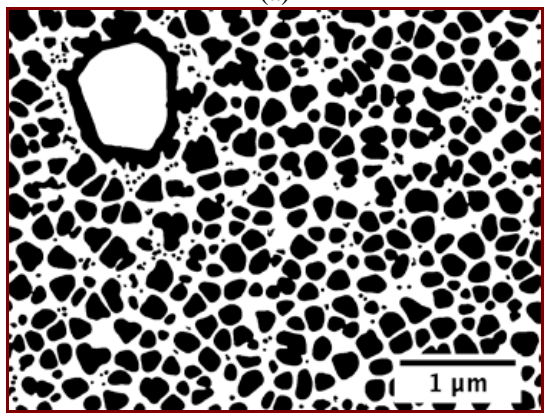

(b)

Figure 6. Thresholded images of the gamma prime after image processing from the (a) un-aged condition (b) dual aged condition

The $\gamma^{\prime}$ volume fraction of the un-aged LSHR was measured to be $44 \pm 2 \%$, where $42 \%$ comprised of secondary precipitates and $2 \%$ tertiary. The average equivalent diameter of the secondary and tertiary $\gamma$ was $175 \pm 46$ and $29 \pm 17 \mathrm{~nm}$, respectively. The $\gamma^{\prime}$ 
volume fraction in the dual aged material was found to be $48 \pm$ $3 \%$, where $45 \%$ were secondary and $3 \%$ tertiary. The average equivalent diameter of the secondary and tertiary thi s microstructural condition was $179 \pm 54$ and $36 \pm 17 \mathrm{~nm}$, respectively. Further research aimed at improving the accuracy of measuring the $\gamma^{\prime}$ is currently being performed.

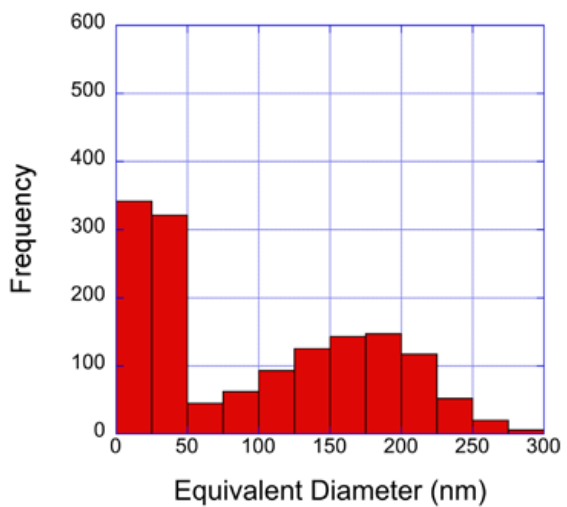

(a)

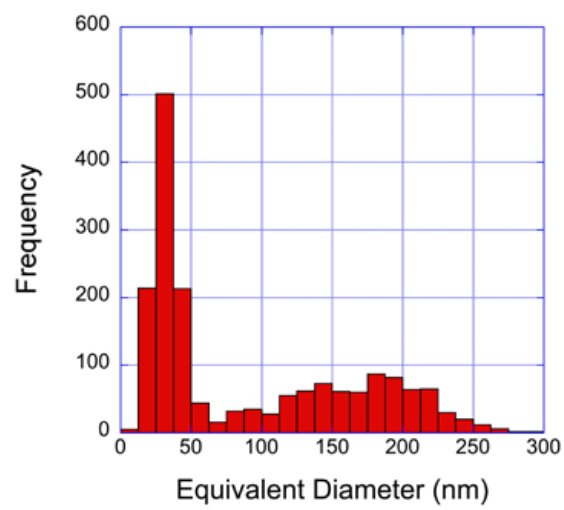

(b)

Figure 7. (a) Histogram of un-aged microstructure (b) histogram of dual aged microstructure

Figure 7(a) and 7(b) display histograms for thedjstributions (including both secondary and tertiary precipitates) for each microstructural condition and further amplify the difference in the $\gamma^{\prime}$ population after aging. The histograms clearly show a bimodal distribution in both microstructures. However, independent assessments of the growth and coarsening of the gamma prime can not made at this time. To fully address the coarsening behavior of the alloy, the stability of the $\gamma$ volume fraction would have to be observed through longer aging exposures (at a given temperature) [17].

Mechanical Testing of Unaged Microstructure

The room temperature $0.2 \%$ offset yield strength of the un-aged sample was measured to be $1003 \mathrm{MPa}$, with a total strain of $2.98 \%$ experienced by the sample. Figure 8 displays the strain map output from the VIC-2D software as time elapsed during the test. The largest amount of strain was detected in the center of the sample at $3.11 \%$. For the sake of simplicity, three different locations on the sample were chosen to display changes in the accumulated strain over time.

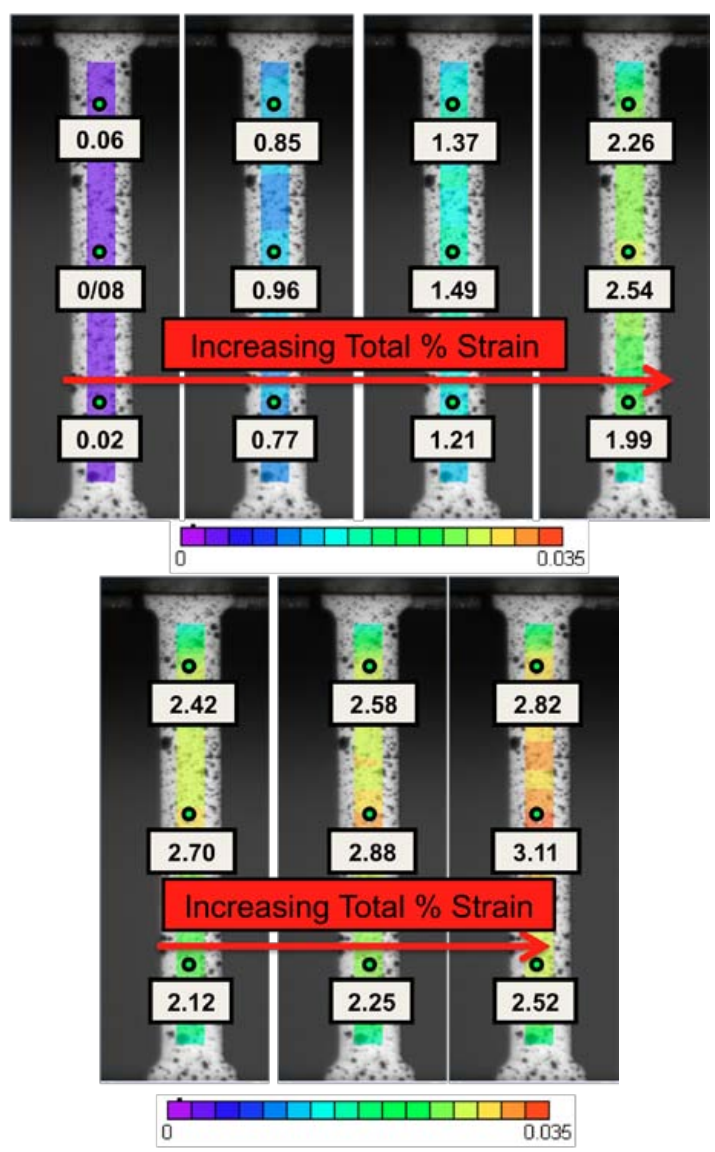

Figure 8. VIC-2D strain maps of the un-aged material in different instances of time. The levels of strain are as indicated, with the largest strains accumulating in the center of the sample $3.11 \%$

Characterization of Unaged LSHR After Testing

The highly localized region of un-aged sample was further investigated with EBSD measurements. Figure 10 displays the grain reference orientation deviation (GROD) and IPF maps overlaid with the image quality (IQ) parameter of the localized region after the test. The GROD map measures the angular deviation of each pixel within a grain with respect to the grain's reference orientation [18], in this case, its average orientation. A color-scale is then applied to the spectrum of calculated angular deviations allowing one to visualize the local crystal rotations within each grain.

However, it should be noted that care must be exercised when forming GROD maps. For instance, before these maps are constructed, the user must specify the critical misorientation and minimum pixel size that define a grain in the TSL software. An appropriate choice of critical misorientation is crucial to distinguishing strain localization from low-angle boundaries. 
Furthermore, the minimum pixel size must be carefully selected as it can depend on the step size chosen for the scan, i.e. the number of pixels allotted for each grain. To minimize such complications, the scan and software parameters were consistent for each microstructure studied.

With proper caution, a GROD map can reveal strain localizations. However, its effectiveness may be increased by overlaying a map of the IQ parameter. The IQ parameter is a measure of Kikcuhi band intensity observed at each data point [19] and can reveal the presence of lattice distortions, in particular those due to strain in the sample [18]. When the lattice is strained, the ideal diffracting conditions are no longer present and thus these areas appear darker in the image quality map.

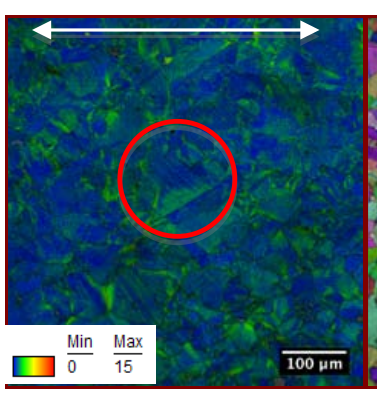

(a)

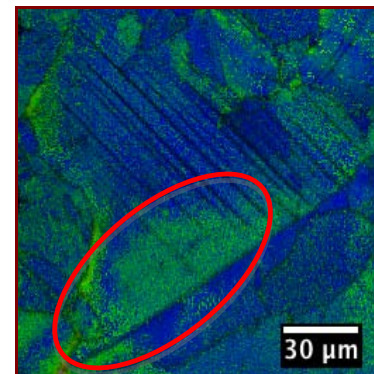

(c)

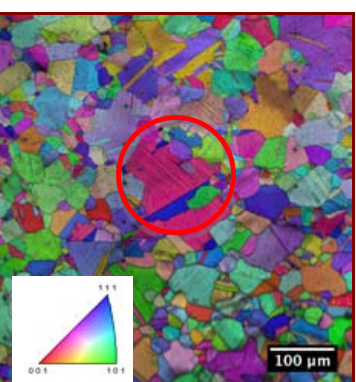

(b)

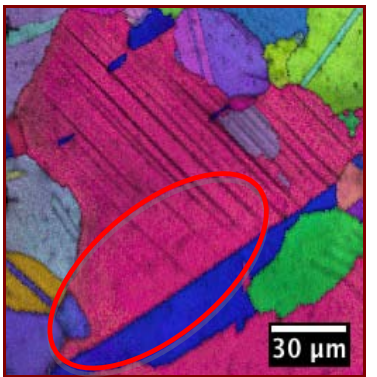

(d)
Figure 9. (a) GROD map with IQ parameter, the double sided arrow indicates the loading direction (b) Corresponding IPF map overlaid with the IQ (c) GROD of a grain with a large amount of misorientation at grain boundary (circled) (d) complimentary IPF map selected grain displaying diffuse slip traces at the highly misoriented grain boundary

The IPF maps in Figure 9 display apparent slip traces induced from the tensile testing as dark linear features within the grains. These linear features are also recognizable in the GROD maps. In this instance they appear as additional sources of lattice misorientation (shown in Figure 9(c)). An interesting phenomenon was observed such that as the slip traces approach several grain boundaries, the linear features attributed to poor image quality become more diffuse (shown in Figure 9(d)). This same region appears to be highly misoriented relative to the rest of the grain (shown in Figure 9(c)). This diffuse IQ is observed in several areas of the microstructure. According to Courtney [20] some grains in the microstructure are more favorably oriented than others for plastic flow, i.e. more favorable for the movement of dislocations. This produces strain displacements across the grain boundary, which leads to strain gradients within individual grains. Thus, the dislocation density in the regions of the grain boundary is greater than the interior [20]. However, further investigation of the highly misoriented grain boundaries in the microstructure is required to confirm the actual deformation activity present.

A FIB TEM sample was extracted from a population of slip traces in the central strained region of the tensile specimen. Zone axis BF STEM imaging was performed on the $<110>$ zone. Figure 10 displays the observed dislocation activity.

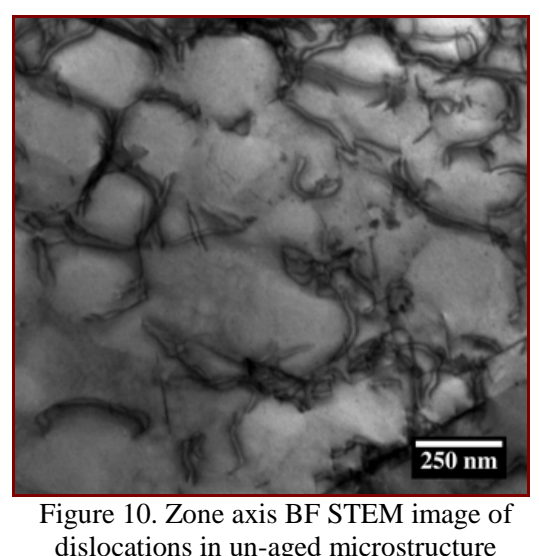

The majority of the dislocations appeared to be within the $\gamma$ channels of the larger $\gamma^{\prime}$ precipitates. It is accepted that that the observed deformation is a function of temperature, strain rate and total strain. Since the mechanical performance of the microstructure is of value, the activity observed in the un-aged condition will be contrasted with all future microstructure conditions of interest. In this case, the next microstructure to be evaluated was the coarse grain microstructure subject to the dual aging heat treatment.

\section{Mechanical Testing of Dual Aged LSHR}

A room temperature tensile test was performed on the dual aged coarse grain microstructure at a strain rate of $8.3 \times 10^{-5} \mathrm{~s}^{-1}$. The $0.2 \%$ offset yield strength was determined to be $1105 \mathrm{MPa}$ and the total strain the sample experienced was $2.80 \%$. Figure 11 shows the strain maps generated by VIC-2D as time elapsed in the test. Unlike the un-aged sample, this specimen displayed three regions of highly localized strain in the gage section. 

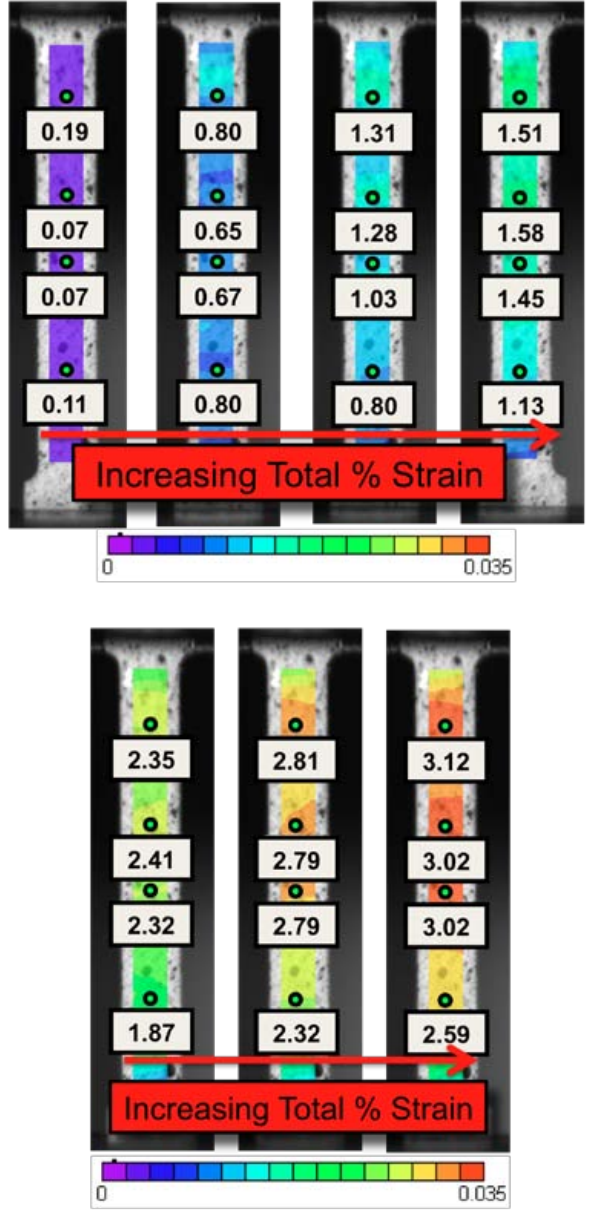

Figure 11. Strain mapping of dual aged microstructure condition and increasing levels of strain as time elapsed

Characterization of Dual Aged LSHR After Testing

For the sake of comparison to un-aged condition, the initial investigation of the dual aged microstructure focused on the central region of the gage. This region presented a localized strain of $3.02 \%$, which was still higher than measured bulk strain at $2.80 \%$. Figure 12 displays the corresponding GROD and IPF maps. Like the un-aged sample, slip traces are observed throughout the scanned area.

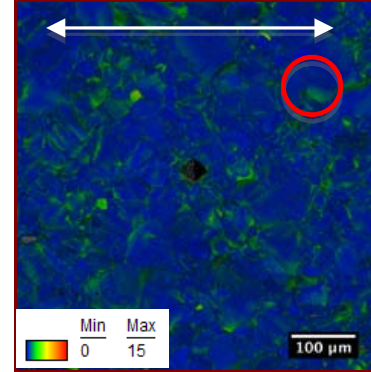

(a)

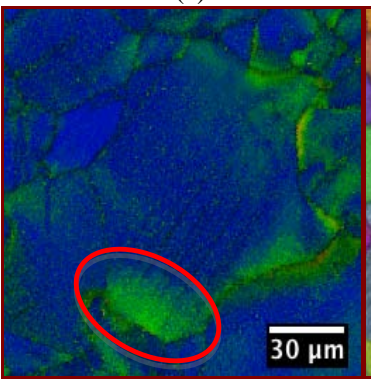

(c)

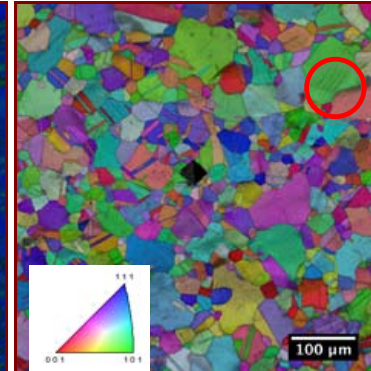

(b)

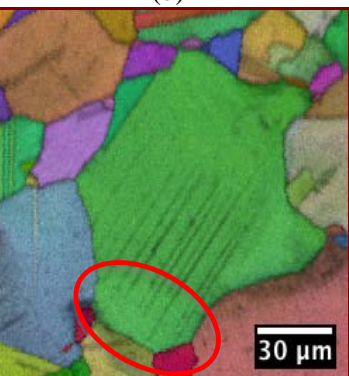

(d)
Figure 12. EBSD of dual aged microstructure (a) GROD map overlaid with IQ parameter, the double sided arrow indicates the loading direction (b) Corresponding IPF map IQ parameter (c) Misorientation at the grain boundary (d) IPF map showing diffuse slip traces approaching grain boundary

Similar to the un-aged condition, in several locations in the microstructure the image quality parameter becomes more diffuse as the misorientation at the grain boundary increased, as shown in Figure 12(c) and 12(d). As previously mentioned, this may be due to a preferred slip orientation within the grain. However, further investigation is required to confirm the precise deformation behavior. Figure 13 displays a side-by-side comparison of the misorientations in each microstructure condition.

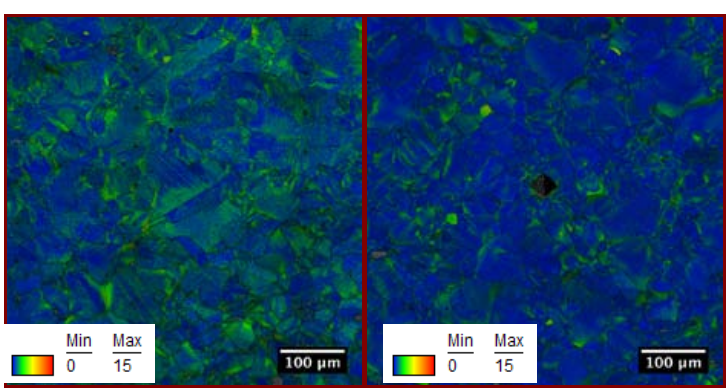

(a)

(b)

Figure 13. Direction comparison of misorientations after tensile testing for (a) unaged and (b) dual aged microstructures

Figure 13 shows that there were more detected misorientations in the unaged microstructure than the dual aged in the central section of the gage. This is an interesting observation that is still not understood. A FIB foil was extracted from slip traces within a 
grain interior in the dual aged condition. The $<110>$ zone axis $B F$ STEM image of the dislocation activity is displayed in Figure 14.

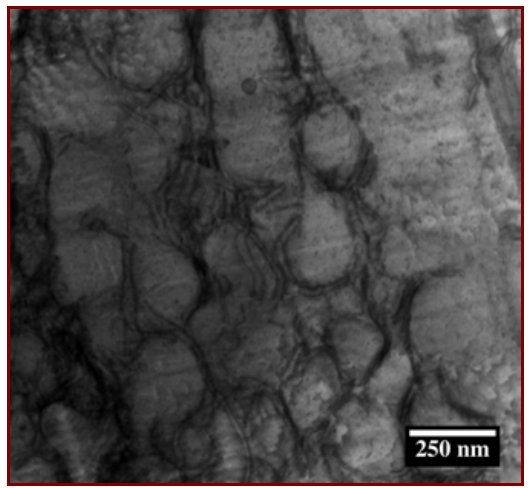

Figure 14. BF STEM image of dual aged microstructure

Similar to un-aged condition, the majority of the dislocation activity in this zone axis appears to be collected around $\gamma^{\prime}$ in the $\gamma$ channels. This is an interesting observation, considering the yield strength of the dual aged specimen is approximately $100 \mathrm{MPa}$ higher than the un-aged condition. This further illustrates that there are several microstructural features that contribute to the strength of nickel base superalloys.

\section{Summary and Conclusions}

Coarse grain specimens were extracted from the rim region of an LSHR turbine disk. The effects of a two-step aging sequence were investigated using high resolution scanning electron microscopy and transmission electron microscopy. Bimodal $\gamma^{\prime}$ precipitate populations were observed in each microstructure of interest. Stereological measurements of the bimodal microstructures showed a small quantitative increase in the precipitate volume fraction and average equivalent diameter as a function of aging.

Digital image correlation performed on the subscale tensile specimens successfully showed intense strain localization in the un-aged and dual aged conditions. However, the specimen with the dual aging heat treatment displayed three regions of highly localized strain where the un-aged sample appeared to have one. In this study, the central regions of localized strain intensity in each microstructure were examined for microstructural differences. The additional strain localizations in dual aged condition are the subject of a further investigation.

EBSD measurements of both microstructures showed that slip traces in particular grains become less detectable as the misorientation increased at a grain boundary. This effect is likely due to a favorable slip orientation within grains, which enables enhanced dislocation activity, but this needs to be confirmed with future research. EBSD measurements of the two microstructures displayed more widespread misorientation in the un-aged microstructures versus the dual aged microstructure. Bright field STEM dislocation imaging was pursued to observe the dislocation activity within the highly localized strained regions of both samples. Both microstructures showed dislocations accumulating within the $\gamma$ channels of the secondary $\gamma^{\prime}$. Overall, both microstructures presented similar deformation features, but the dual aged condition provided a higher yield strength. Additional research will be performed to determine exact microstructural features that contribute to this performance.

\section{Acknowledgements}

The authors would like to gratefully acknowledge the support of Air Force Research Laboratories under the STW21 II contract FA9550-09-1-0014 (AFSOR).

\section{References}

1. J. Gayda, T.P. Gabb, P.T. Kantzos, "The Effect Dual Microstructure Heat Treatment on an Advanced NickelBase Disk Alloy”, Superalloys 2004, ed. K.A. Green, et al (Warrendale, PA; The Minerals, Metals and Materials Society, 2004), 323-329.

2. T.P. Gabb, J. Gayda, J. Telesman, P.T. Kantzos, "Thermal and Mechanical Property Characterization of the Advanced Disk Alloy LSHR” (Report NASA/TM2005-213645, National Aeronautics and Space Administration, 2005)

3. J. Gayda, D.U. Furrer, "Dual Microstructure Heat Treatment,” Advanced Materials \& Processes, v 161 (7) (2003), 36-39.

4. R.W. Kozar, A. Suzuki, W.W. Milligan, J.J.Schirra, M.F. Savage, T.M. Pollock, "Strengthening Mechanisms in Polycrstalline Multimodal Nickel-Base Superalloys," Metallurgical and Materials Transactions A, v 40 (7) (2009), 1588-1603.

5. R.R. Unocic, G.B.Viswanathan, P.M. Sarosi, S. Karthikeyan, J. Li, M.J. Mills, "Mechanisms of Creep Deformation in Polycrystalline Ni-base Superalloys," Materials Science and Engineering A, v 483-484 (1-2C) (2008), 25-32.

6. G.B. Viswanathan, S. Karthikeyan, P.M. Sarosi, R.R. Unocic, M.J. Mills, "Microtwinning during intermediate temperature creep of polycrystalline Ni-based superalloys: mechanisms and modelling," Philosophical Magazine, v 86 (29-31) (2006), 4823-4840.

7. G.B. Viswanathan, P.M. Sarosi, M.F. Henry, D.D. Whitis, W.W. Milligan, M.J. Mills, "Investigation of creep deformation mechanisms at intermediate temperatures in Rene' 88 DT," Acta Materialia, v 53 (10) (2005), 3041-3057.

8. J. Gayda, P. Kantzos, "High Temperature Burst Testing of a Superalloy Disk With a Dual Grain Structure" (Report NASA/TM-2004-212884, National Aeronautics and Space Administration, 2004).

9. T.P. Gabb, J.Gayda, J. Telesman, A. Garg, “The Effects of Heat Treatment and Microstructure Variations on Disk Superalloy Properties at High Temperature", Superalloys 2008, ed. R.C. Reed et al (Warrendale, PA; The Minerals, Metals and Materials Society, 2008), 121-129.

10. A. Soula, Y. Renollet, D. Boivin, J.L. Pouchou, D. Locq, P. Caron, Y. Brechet, "Grain Boundary and Intragranular Deformations During High Temperature Creep of a PM Nickel-Based Superalloy”, Superalloys 2008, ed. R.C. Reed et al (Warrendale, PA; The Minerals, Metals and Materials Society, 2008) 387-394. 
11. Peterson, B.H., "A Combinatorial Approach to the Development of a Creep Resistant Beta Titanium Alloy” (Ph.D. thesis, The Ohio State University, 2008) 49-57.

12. M.A. Sutton, "Digital Image Correlation for Shape and Deformation Measurements", Springer Handbook of Experimental Solid Mechanics, ed. W. Sharpe, Springer, 2008, 565-600.

13. Michael A. Sutton, Jean-Jose Orteu, Hubert W. Schreier, Image Correlation for Shape, Motion and Deformation Measurements: Basic Concepts, Theory and Applications (New York, NY: Springer, 2009)

14. E.J. Payton, P.J. Phillips, M.J. Mills, "Semi-automated characterization of the gamma prime phase in Ni-based superalloys via high-resolution backscatter imaging” Materials Science and Engineering A, v 527 (10-11) (2010), 2684-2692.

15. P.J. Phillips, M.C. Brandes, M.J. Mills, M. De Graef, "Diffraction Contrast STEM of dislocations: Imaging and Simulations", Ultramicroscopy, v 111 (9-10) (2011), 1483-1487.

16. Kuhr, S.J., Viswanathan, G.B., Tiley, J., Fraser, H.F., Unpublished research, Materials Science Department, The Ohio State University, 2012.

17. J. Tiley, G.B. Viswanathan, R. Srinivasan, R. Banerjee, D.M. Dimiduk, H.L. Fraser, "Coarsening kinetics of c0 precipitates in the commercial nickel base Superalloy Rene' 88 DT”, Acta Materialia, v 57 (8) (2009), 25382549.

18. TSL OIM Analysis [Computer Software], 2007. TexSEM Laboratories.

19. S.R. Claves, A. Deal, "Orientation Dependence of EBSD Pattern Quality”, Microscopy and Microanalysis, v 11 (S02) (2005), 514-515.

20. Thomas H. Courtney, Mechanical Behavior of Materials (New York, NY: McGraw Hill, 2000), 140169. 